\title{
Identification of Pyrotechnic Shock Sources for Shear Type Explosive Bolt
}

\author{
Yanhua Li, ${ }^{1}$ Shihui Xiong, ${ }^{1}$ Yuan Li, ${ }^{1}$ Xiaogang Li, ${ }^{1}$ Yuquan Wen, \\ Huina $\mathrm{Mu},{ }^{1}$ and $\mathrm{Zhiliang} \mathrm{Li}^{2}$ \\ ${ }^{1}$ State Key Laboratory of Science and Technology, Beijing Institute of Technology, Beijing 100081, China \\ ${ }^{2}$ CNGC Xian Qinghua, North Special Energy Group Co. Ltd., Xian 710025, China
}

Correspondence should be addressed to Yuquan Wen; wyquan@bit.edu.cn

Received 16 August 2017; Accepted 27 November 2017; Published 14 December 2017

Academic Editor: Salvatore Russo

Copyright (C) 2017 Yanhua Li et al. This is an open access article distributed under the Creative Commons Attribution License, which permits unrestricted use, distribution, and reproduction in any medium, provided the original work is properly cited.

\begin{abstract}
To identify the shock generated sources of shear type explosive bolt, five kinds of explosive bolts with different states, including high preload, low preload, no preload, no piston shear, and only propellant combustion, were designed. The decoupling of four kinds of shock sources, including the collision of propellant combustion, piston shear weak surface, end of piston stroke, and preload release, was realized. Their acceleration responses at $15 \mathrm{~cm}$ from the shock source center on $60 \mathrm{~cm} \times 60 \mathrm{~cm} \times 1 \mathrm{~cm}$ equivalent aluminum plate were measured by using the piezoresistive accelerometer. On this account, the relative contribution values along with frequency and the mean relative values without regard to frequency of various sources were calculated in high and low preload. Results show that the characteristics of different shock sources are different, and the shock induced by propellant combustion is mainly caused by high frequency, while piston shear and piston impact are concentrated in medium and low frequency. In the high preload condition, the preload release is major contributor to the overall shock response output, accounting for approximately $57.51 \%$, while the contributions of the three shock sources of propellant combustion, piston shear, and piston impact are equivalent, but they are significant contributors in the low preload condition. This study provides design reference to reduce the pyrotechnic shock of shear type explosive bolt.
\end{abstract}

\section{Introduction}

Explosive bolt is a kind of simple, high reliable, and efficient pyrotechnic release devices, widely used in aerospace, missile weapons, and underwater launch system, such as booster rockets separation, stage separation, fairing separation, and the release of external tanks $[1,2]$. Various designs of explosive bolts exist and can be categorized into high explosive type and pressure type [3-8]. To break the bolts, high explosive type utilizes the shock wave generated by detonation, while pressure type utilizes the high pressure generated by explosion and combustion in the cavity. The pressure type is prevalent because of the advantages: that is, it has relatively lighter shock on installation structure and does not produce fragments and product leakage, namely, noncontamination, and the shear type explosive bolt belongs to this type.

As with various pyrotechnic devices, the action of explosive bolt inevitably induces high frequency and high amplitude transient shock, known as pyroshock that can easily cause failures in nearby electronic, optics, relay, and magnetic components and finally leads to whole space mission failure [9-11]. To mitigate damage from pyroshock, most previous studies during the past half century have focused on pyroshock propagation and damage evaluation with either numerical or experimental methods, and numerous test standards [11-14] and technical reports $[15,16]$ are published, while relatively few studies have reported on pyroshock generation. Past shock preventive measures also mostly focus on isolation from the shock propagation path and instrument installation [17]. As the application of lightweight space structure, such as small satellites and deep space probes, the above two methods are limited. Therefore, a general trend appears to reduce the shock level generated by separation device itself.

Han team from South Korea [4, 18-21] has deeply analyzed and researched the separation mechanism of the "ridgecut" explosive bolt by AUTODYN since 2004. They have 


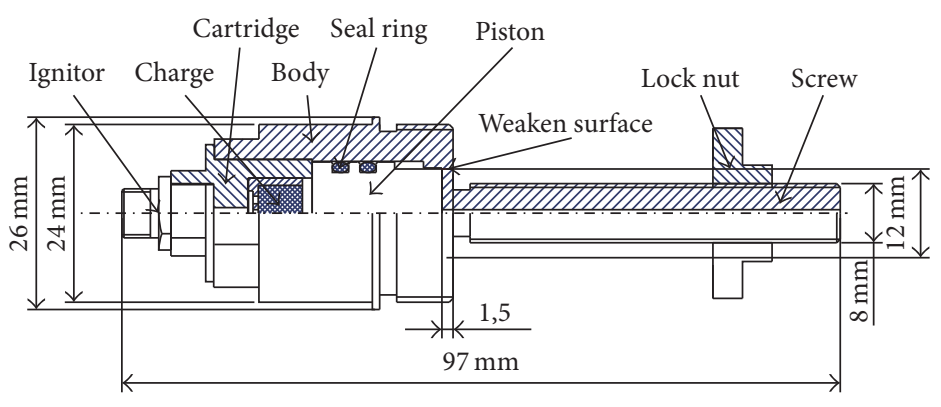

(a)

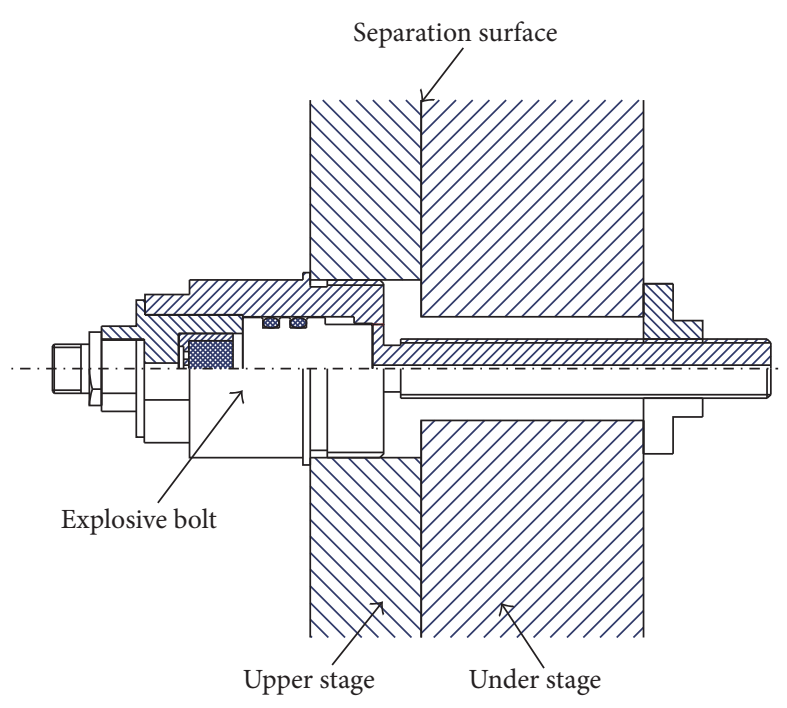

(b)

FIGURE 1: Structural schematic diagrams of shear type explosive bolt: (a) structure composition and (b) installation diagram.

analyzed effects of charge amount, the gap between bolt body and the fixture, ridge angle, and ridge position on the separation feature and proposed three improvements, which reduce the output shock from blocking or dissipating the shock wave propagation of high explosives without considering the shock response caused by preload. In 2015, Huang et al. [22] analyzed the influence of explosive bolt preload on output shock by LS-DYNA numerical simulation. The results show that the preload within a certain range had less effect on the shock response in middle and far filed. Compared with the above high explosive type bolts, pressure type bolts are more complex due to the movement of internal components, and the research is rather little. In 2011, Ye and Yan [23] made the impulse of pyrotechnic separation blot reduce from $1.667 \mathrm{~N} \cdot \mathrm{S}$ to $0.4 \mathrm{~N} \cdot \mathrm{S}$ by the two technical approaches of optimizing structure size and charge amount. In 2015, Liu et al. [24] extracted the velocity and acceleration of the body and piston by the LS-DYNA and calculated out the impact force and impulse of explosive bolt. However, this was not the total response output of the bolt.

In this study, the possible sources of the shock output are obtained by the analysis of the separation mechanism of the shear type explosive bolts. Five kinds of explosive bolts in different operating conditions are designed to carry out shock output tests; meanwhile, the individual shock response from each source compared with the overall SRS is calculated.

\section{Structure and Principle}

The shear type explosive bolt under study is shown in Figure 1(a). It is mainly composed of ignitor, pyrotechnic component (including main charge and charge cartridge), piston, seal ring, and body component (including bolt body, screw, and lock nut). The material of body and piston is, respectively, $1 \mathrm{Cr} 11 \mathrm{Ni} 2 \mathrm{~W} 2 \mathrm{MoV}$ heat-resistant steel and 60Si2MnA spring steel. There is a preset weaken surface in explosive bolt body which is able to withstand a certain load. In the connection state, bolt body is screwed on the upper stage, and the screw passes through the two connected stages which are reliably connected by the lock nut with a certain preload, as shown in Figure 1(b).

The separation process of shear type explosive bolt is shown in Figure 2. When the target body needs to be separated, ignitor is powered to initialize and then it ignites the main charge (Figure 2(a)), producing high temperature and high pressure gas to push the piston shear weak surface along with preload release (Figure 2(b)). When the weak surface is completely sheared, the piston with the screw speeds up to move forward and then impacts the body (Figure 2(c)) to stop motion; then the screw with lock nut leaves from piston to complete separation (Figure 2(d)). Two seal rings seal the combustion products in the body cavity to realize noncontamination separation.

The physical diagram of shear type explosive bolt is shown in Figure 3. It can be seen that the bolt body has no obvious deformation and sheared surface is neat without redundant fragments, which means that the shear explosive bolts achieve perfect and clean separation.

According to the separation mechanism of shear type explosive bolt (see Figure 2), the pyroshock generation of the explosive bolt may be caused by four main events: (1) propellant combustion, (2) piston shear weaken surface, (3) bolt preload release, and (4) internal piston impact. To identify their characteristics, special tests need to be designed.

\section{Pyrotechnic Shock Measurement of Shear Type Explosive Bolts}

Pyroshock could be theoretically characterized as transient force, strain, velocity, and acceleration. The measurement methods have developed from the traditional contact point 


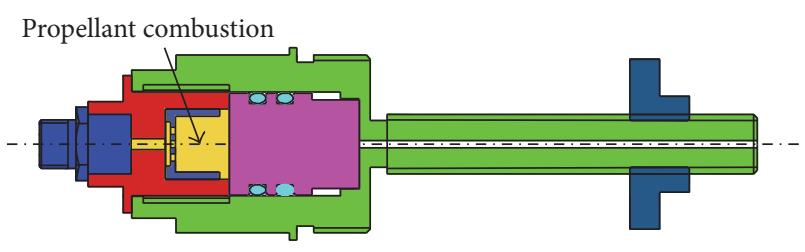

(a)

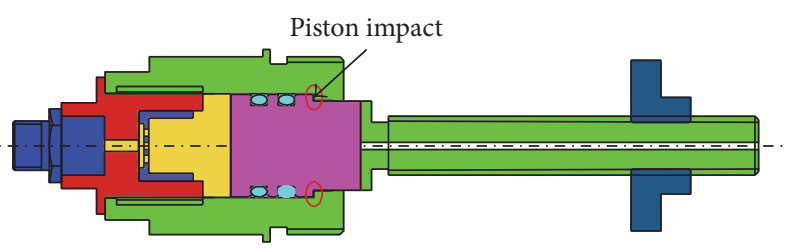

(c)

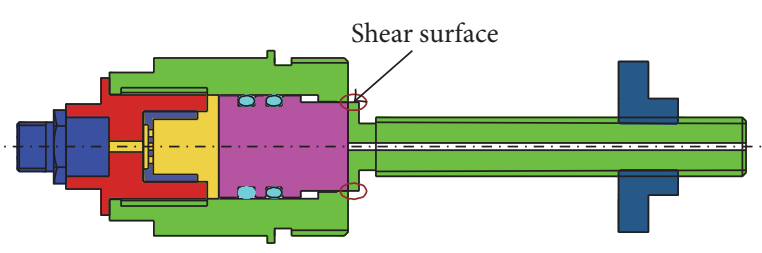

(b)

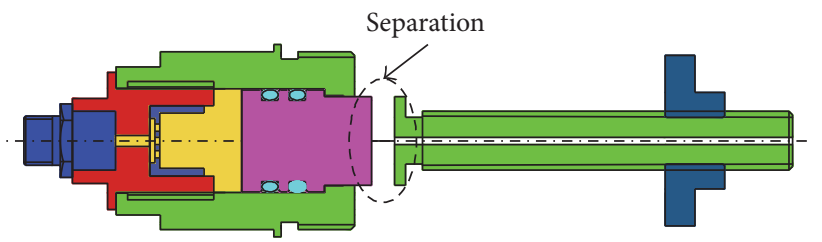

(d)

FIGURE 2: Schematic diagrams of separation mechanism for shear type explosive bolt: (a) step 1, (b) step 2, (c) step 3, and (d) step 4.

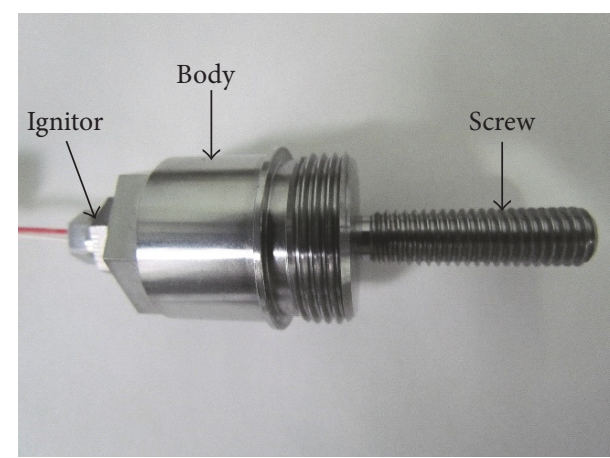

(a)

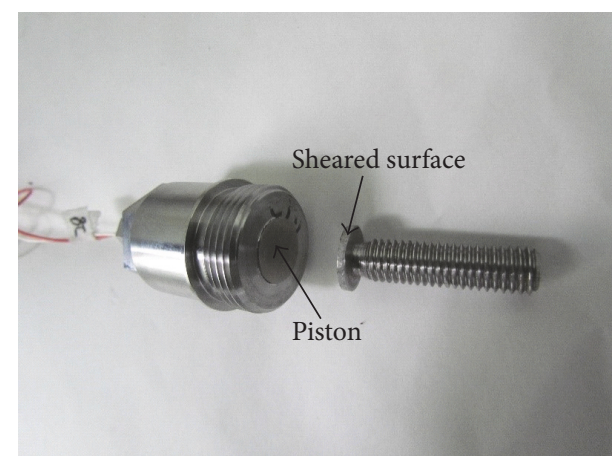

(b)

FIGURE 3: Pictures of shear type explosive bolts: (a) before separation and (b) after separation.

measurement to the modern noncontact full area measurement, such as the strain gauge, accelerometer, laser Doppler vibrometer (LDVs), laser Doppler accelerometer (LDAc), and ultra-high speed scanning camera [25]. Considering convenience and cost, the study uses the accelerometer to measure the shock acceleration of shear type explosive bolt and then calculates its response spectrum.

3.1. Experimental Setup. The shock output of the explosive bolts is characterized with a $60 \mathrm{~cm} \times 60 \mathrm{~cm} \times 1 \mathrm{~cm}$ aluminum plate suspended from bungees. The explosive bolt is screwed on the aluminum block $(4 \mathrm{~cm} \times 4 \mathrm{~cm} \times 1 \mathrm{~cm})$ and the connected preload is applied by the lock nut in the other side of bench. In order to accurately load tensile force and record its release process, a piezoelectric circle force sensor is placed between lower plate and lock nut. To record shock response of explosive bolt on the test bench, the $\mathrm{PCB}$ piezoresistive accelerometer (Model 3501B1260KG) is screwed on the bench at $15 \mathrm{~cm}$ from the center of explosive bolt. Figures 4 and 5, respectively, show the test setup schematic and test scene.

Its measure principle: the ignition control box gives the ignition signal and ignites the igniter; meanwhile, the data acquisition instrument receives the trigger signal given by the ignition control box and starts to work. Then, explosive bolt begins action and releases screw, signal conditioner amplifies and filters the signal transmitted from force sensor which is outputted to the data acquisition instrument, and bolt preload curve is obtained. At the same time, the shock generated by explosive action transmitted to accelerometer through aluminum plate, and the acceleration signal is displayed on the acquisition instrument through the signal conditioner.

3.2. Instrumentation and Data Acquisition. The MIL-STD$810 \mathrm{G}$ [12] requires that accelerometer for pyroshock measurement should meet the following rules: (1) transverse sensitivity of less than or equal to $5 \%,(2)$ an amplitude linearity within $10 \%$ from $5 \%$ to $100 \%$ of the peak acceleration amplitude required for testing, and (3) a flat frequency response within \pm 10 percent across the frequency range $10-20,000 \mathrm{~Hz}$. The performance parameters of PCB 3501B260KG accelerometer are shown in Table 1. As shown in Table 1, the PCB 3501B260KG accelerometer meets the shock measurement requirements.

In the proshock shock test, it often happens that the high frequency shock is rolling off low frequency. Two common methods are usually used to prevent aliasing: (1) using a 
TABLE 1: Performance parameters of accelerometer.

\begin{tabular}{|c|c|c|c|c|c|c|}
\hline $\begin{array}{l}\text { Model } \\
\text { number }\end{array}$ & $\begin{array}{l}\text { Transverse } \\
\text { sensitivity }\end{array}$ & $\begin{array}{c}\text { Amplitude } \\
\text { (linearity } \pm 10 \% \text { ) }\end{array}$ & $\begin{array}{l}\text { Frequency range } \\
\text { (flatness } \pm 10 \% \text { ) }\end{array}$ & $\begin{array}{l}\text { Resonant } \\
\text { frequency }\end{array}$ & $\begin{array}{c}\text { Electric } \\
\text { insulation }\end{array}$ & Mounting \\
\hline $\begin{array}{l}\text { PCB } \\
3501 B 260 K G\end{array}$ & $\leq 3 \%$ & $\pm 60 \mathrm{~kg}$ & $0-20 \mathrm{kHz}$ & $>120 \mathrm{kHz}$ & Yes & 1/4-28 Stud \\
\hline
\end{tabular}

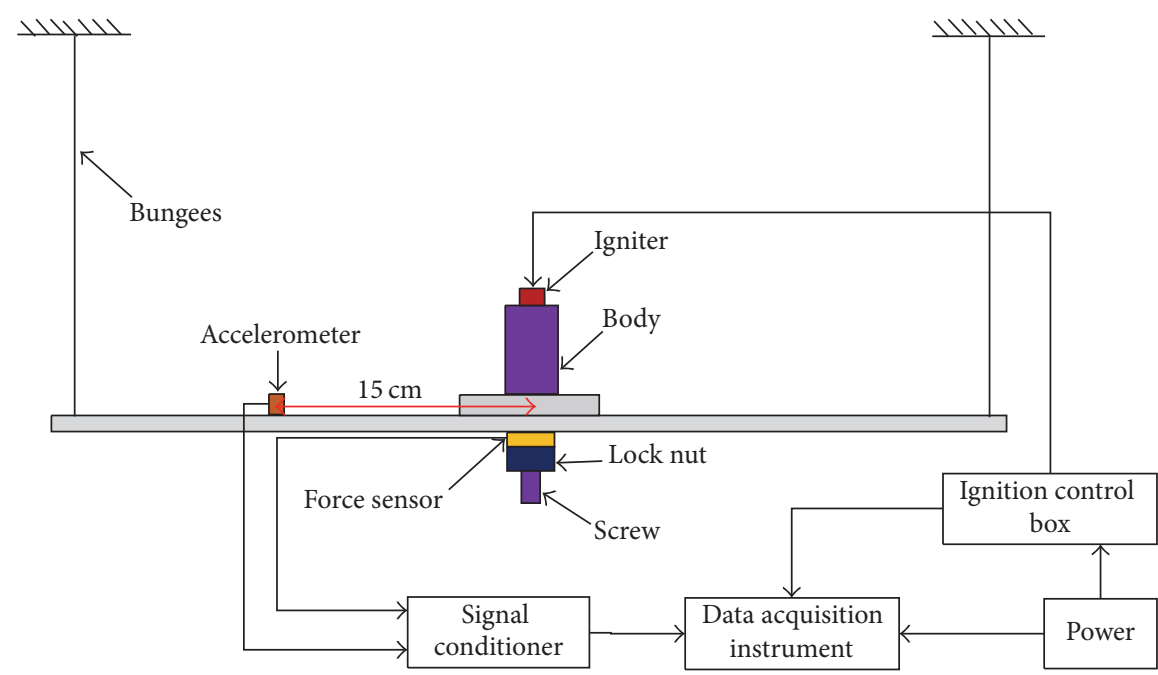

FIGURE 4: Schematic diagram of output shock measurement test platform installation structure for explosive bolts.

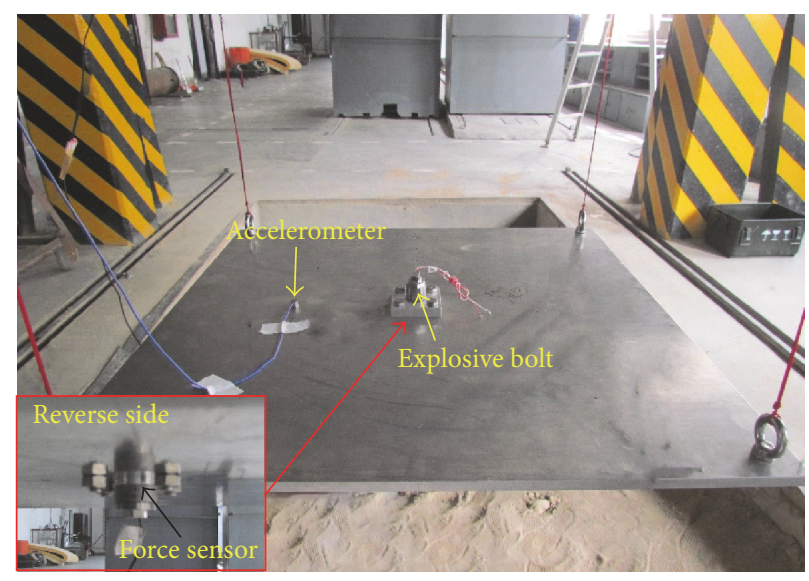

FIGURE 5: Measurement scene of output shock for explosive bolts.

strong analog antialiasing (AAA) filter strategy; although this strategy provides good removal, AAA filters are physically large, expensive, and inherently limited in cutoff capability and phase matching, and (2) oversampling approach, where sampling frequency is much greater than Nyquist frequency, which has the most of quantizing noise distributed outside the interested frequency. In this paper, the second method is adopted where the signal-sampling rate on each channel of acquisition instrument is set to $1 \mathrm{MHz}$.

3.3. Typical Test Data. The shock pulse at different distances from explosive bolt was captured along with the force sensor output and applied bridgewire voltage. The collected typical data is presented in Figure 6 (output shock of the $15 \mathrm{~cm}$ distance from source is shown). When ignitor is powered to initiate, ignite the main charge, and produce high temperature and high pressure gas to push the piston shear weak surface along with preload release, the time is about 0.34 milliseconds. After approximately 0.4 milliseconds, the weak surface is completely sheared because the preload is completely released. The first peak of acceleration is about $11000 \mathrm{~g}$, and the peak time is about 0.52 milliseconds, which happens in the process of preload release. The whole action time is very short which is less than one millisecond, so it is difficult to distinguish contribution of each factor to output shock on the acceleration time history, especially piston shearing process along with preload release. 


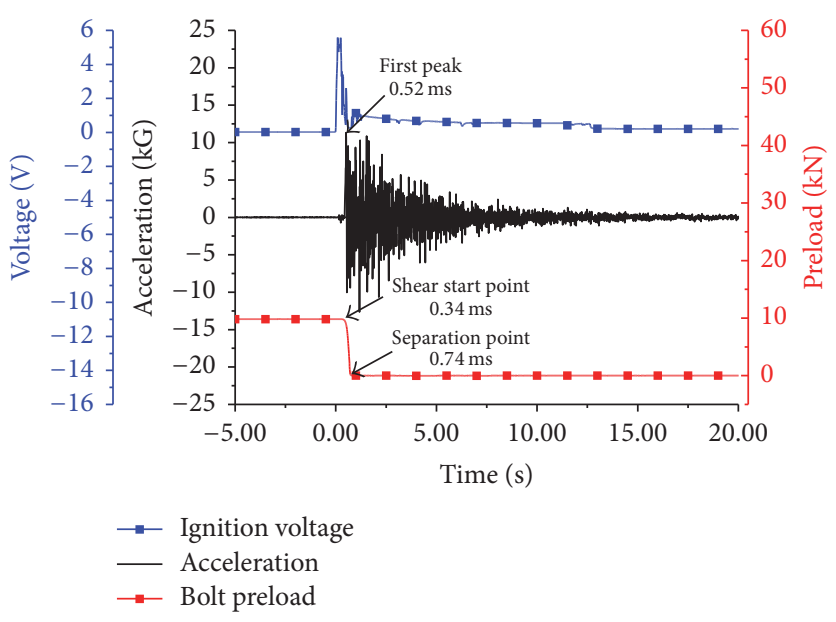

FIGURE 6: Measurement output time history of explosive bolts.

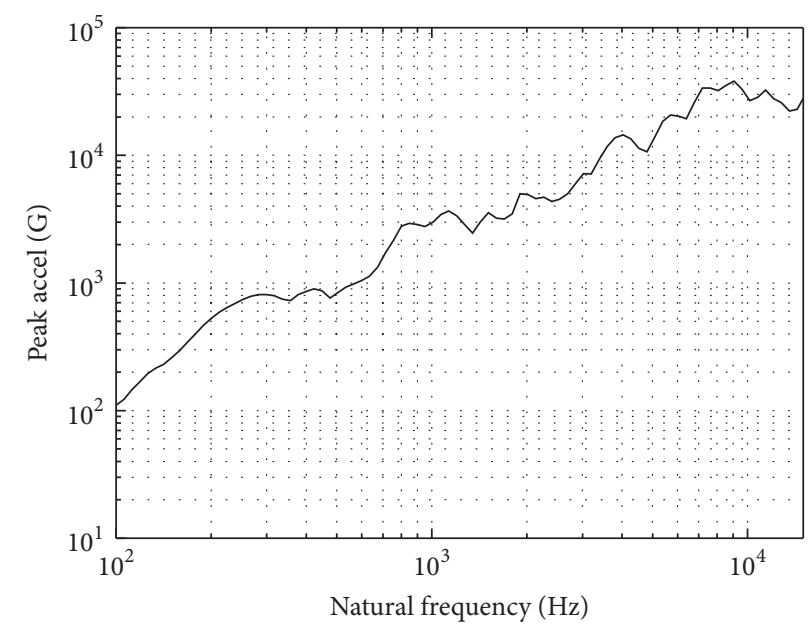

FIGURE 7: Typical shock response spectrum of shear type explosive bolt.

3.4. Shock Response Spectrum. To estimate the damage potential of output shock, the shock response spectrum (SRS) is commonly used, which is peak acceleration response of an array of a single-degree-of-freedom (SDOF) system that has different natural frequencies [10]. The damping of each SDOF system is typically set at 0.05 . Depending on how the peak acceleration is chosen, SRS is categorized as positive, negative, primary, residual, and maxima SRS. Maxima SRS is commonly used for pyroshock quantification which includes all other SRS. From the measured acceleration time history, the maxima SRS is calculated by the improved recursive digital filter method. In order to eliminate high frequency noise, aliasing, and low frequency drift, the 8thorder Butterworth band passing filter between $70 \mathrm{~Hz}$ and $30 \mathrm{kHz}$ is applied to the acceleration time history. This filter has an attenuation slope of $60 \mathrm{~dB} /$ octave, which satisfies the requirement of the NASA standard. From the acceleration, maxima SRS is calculated by using open-source MATLAB codes.

Figure 7 shows the typical shock response spectrum. The results show that the maximum peak acceleration is over
$30,000 \mathrm{~g}$ and the peak frequency is over $8 \mathrm{kHz}$, which is typically high frequency and high amplitude vibration.

\section{Decomposition Analysis of Pyrotechnic Shock Source}

4.1. Experimental Scheme. To identify the four main sources, five different states of explosive bolts are designed and tested by the above pyroshock test bench, and each state tests three samples. The first state is preloaded at $10 \mathrm{kN}$, which captures the shock contribution of propellant combustion, piston shear weaknesses, internal piston impact, and bolt release mechanism. The second state is the same as the first except for different preload of $2 \mathrm{kN}$ to see the influence of the pretension force. The third state is performed, not applying preload to isolate the shock produced by the bolt preload release mechanism. The fourth state whose body is separated in advance captures the shock contribution of propellant combustion wave and internal piston impact. The piston of the last state is fixed by steel washer and glue, so that the 


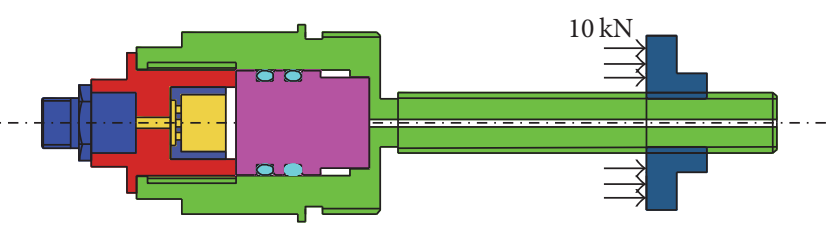

(a)

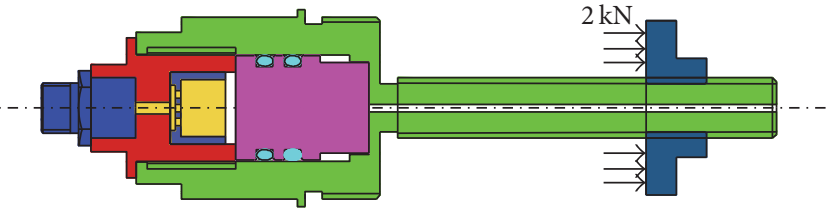

(b)

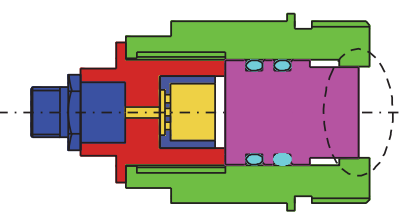

(d)

(c)

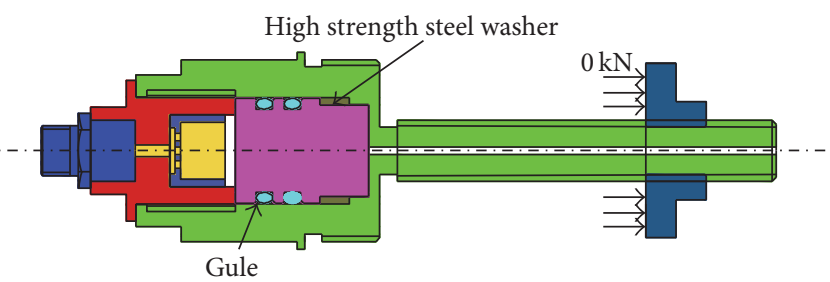

(e)

FIGURE 8: Five different states of explosive bolts: (a) state 1; (b) state 2; (c) state 3; (d) state 4; and (e) state 5.

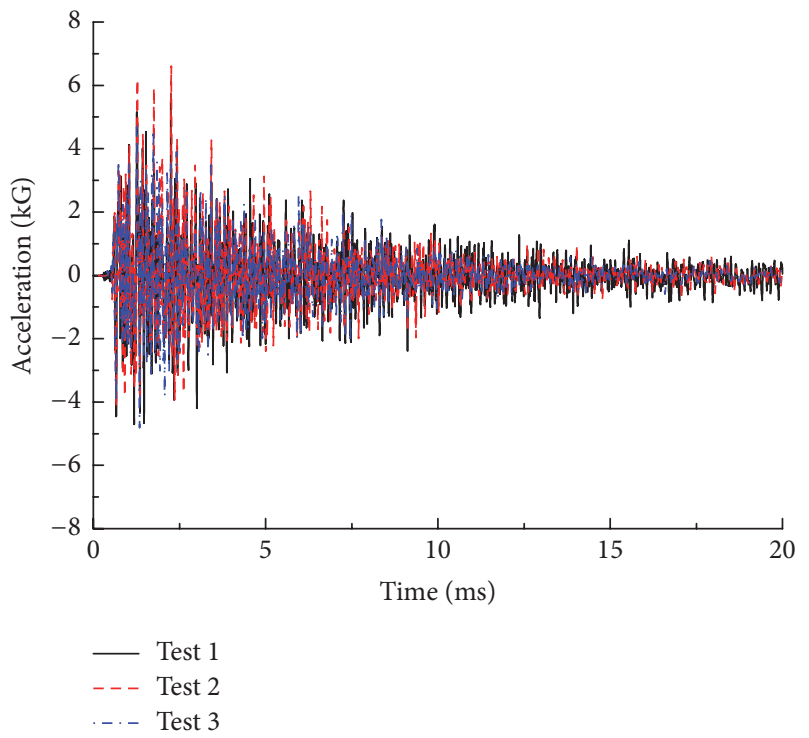

(a)

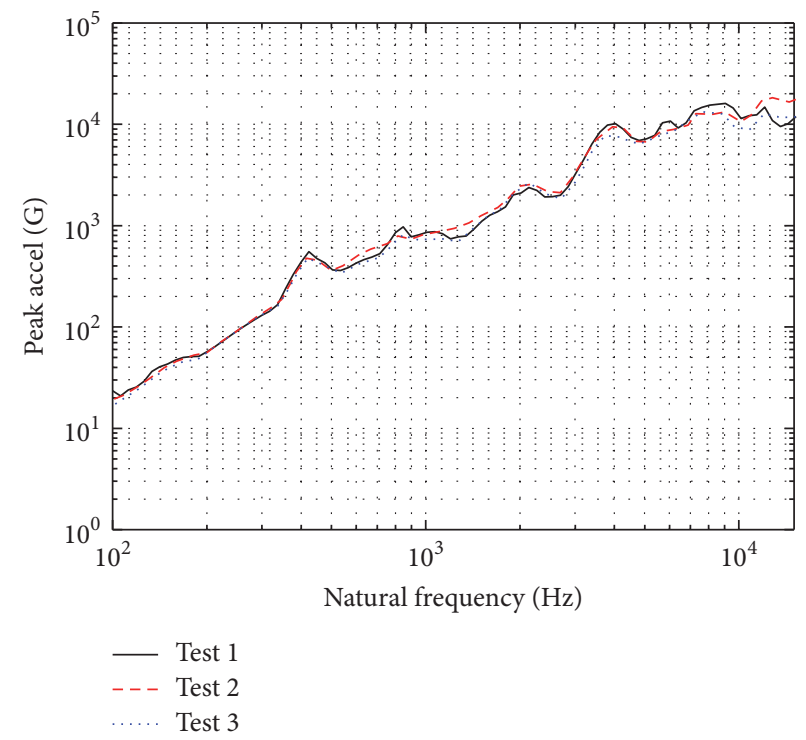

(b)

FIGURE 9: Comparison of three test results of explosive bolts in state 2: (a) acceleration time history and (b) shock response spectrum.

internal pistons no longer move, so the last state test separates out the shock produced by the propellant combustion wave. The experimental schemes are shown in Figure 8.

4.2. Results and Analysis. In order to increase the credibility of the data, each state is tested three samples. The output shock data of state 2 is presented in Figure 9 along with comparative shock response spectra. Due to the randomness of the pyrotechnic incident, there are differences in the acceleration time history and shock response spectrum of the three tests, but the difference is very small, which shows that the samples have good consistency, and the other states are similar. The shock response spectrum of each state is taken as the spectral of three experimental SRS.

The shock response spectra obtained by different state experiments are compared in Figure 10. As can be seen from Figure 10, the result is SRS (state 1) > SRS (state 2) $>$ SRS 


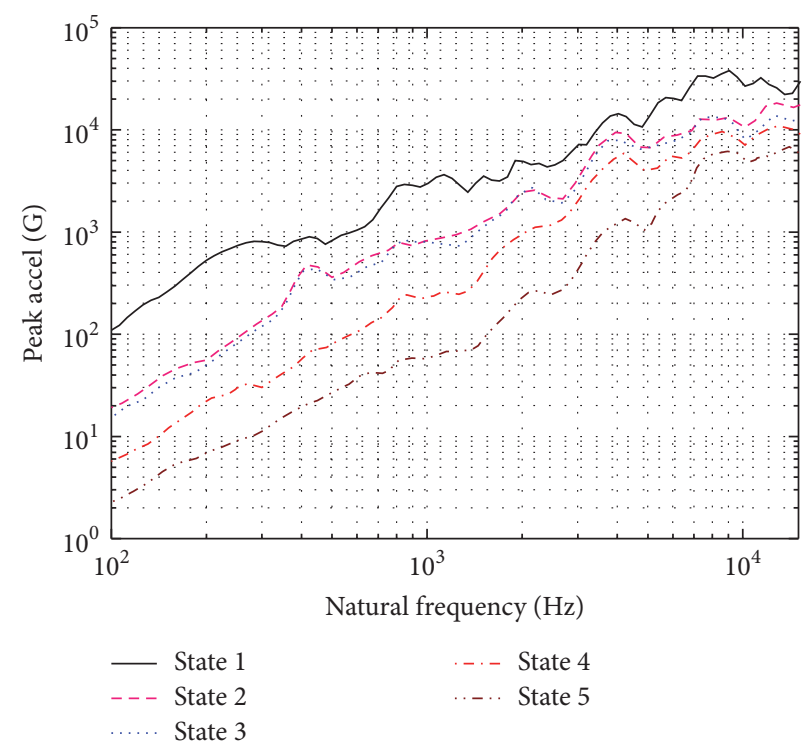

FIGURE 10: SRS comparison of explosive bolts under different states.

(state 3 ) > SRS ( state 4 ) > SRS (state 5) because different state contains different shock sources.

In order to quantify the difference of SRS under different states, a dimensionless coefficient is defined as

$$
\left.E_{a}(f)\right|_{b}=\frac{\operatorname{SRS}_{a}(f)}{\operatorname{SRS}_{b}(f)},
$$

where $\left.E_{a}(f)\right|_{b}$ is the values of $\operatorname{SRS}_{a}(f)$ relative to $\operatorname{SRS}_{b}(f)$ along the frequency range and $\mathrm{SRS}_{b}(f)$ is the base SRS.

According to (1), the relative values of SRS in different states are calculated based on output SRS of explosive bolt with high preload (state 1) or low preload (state 2).

According to the description of experimental scheme, we know the shock sources which every state contains; thus, each even's contribution to the overall SRS in $10 \mathrm{kN}$ preload can be calculated as follows:

$$
\begin{aligned}
& \left.E_{F_{1}}(f)\right|_{\text {state 1 }}=\left.E_{\text {state 1 }}(f)\right|_{\text {state 1 }}-\left.E_{\text {state 3 }}(f)\right|_{\text {state 1 }} \\
& \left.E_{P S}(f)\right|_{\text {state 1 }}=\left.E_{\text {state 3 }}(f)\right|_{\text {state 1 }}-\left.E_{\text {state } 4}(f)\right|_{\text {state 1 }} \\
& \left.E_{P I}(f)\right|_{\text {state 1 }}=\left.E_{\text {state } 4}(f)\right|_{\text {state 1 }}-\left.E_{\text {state } 5}(f)\right|_{\text {state 1 }} \\
& \left.E_{P C}(f)\right|_{\text {state 1 }}=\left.E_{\text {state 5 }}(f)\right|_{\text {state 1 }},
\end{aligned}
$$

where $\left.E_{\text {state } 1}(f)\right|_{\text {state } 1},\left.E_{\text {state } 3}(f)\right|_{\text {state } 1},\left.E_{\text {state } 4}(f)\right|_{\text {state } 1}$, and $\left.E_{\text {state } 5}(f)\right|_{\text {state } 1}$ are, respectively, the relative coefficients of above four states based on state $1 ;\left.E_{P C}(f)\right|_{\text {state } 1}$, $\left.\left.E_{P S}(f)\right|_{\text {state } 1} E_{P I}(f)\right|_{\text {state } 1}$, and $\left.E_{F_{1}}(f)\right|_{\text {state } 1}$ are, respectively, calculated contribution of propellant combustion, piston shear, piston impact, and preload release in high preload state $(10 \mathrm{kN})$.
Similarly, each source's contribution to the overall SRS in $2 \mathrm{kN}$ preload also can be calculated as follows:

$$
\begin{aligned}
& \left.E_{F_{2}}(f)\right|_{\text {state 2 }}=\left.E_{\text {state 2 }}(f)\right|_{\text {state 2 }}-\left.E_{\text {state 3 }}(f)\right|_{\text {state 2 }} \\
& \left.E_{P S}(f)\right|_{\text {state 2 }}=\left.E_{\text {state 3 }}(f)\right|_{\text {state 2 }}-\left.E_{\text {state 4 }}(f)\right|_{\text {state 2 }} \\
& \left.E_{P I}(f)\right|_{\text {state 2 }}=\left.E_{\text {state 4 }}(f)\right|_{\text {state 2 }}-\left.E_{\text {state 5 }}(f)\right|_{\text {state 2 }} \\
& \left.E_{P C}(f)\right|_{\text {state 2 }}=\left.E_{\text {state 5 }}(f)\right|_{\text {state 2 }},
\end{aligned}
$$

where $\left.E_{\text {state } 2}(f)\right|_{\text {state 2 }},\left.E_{\text {state } 3}(f)\right|_{\text {state 2 }},\left.E_{\text {state } 4}(f)\right|_{\text {state 2 }}$, and $\left.E_{\text {state } 5}(f)\right|_{\text {state } 2}$ are, respectively, the relative coefficients of the above four states based on state $2 ;\left.E_{P C}(f)\right|_{\text {state 2 }}$, $\left.\left.E_{P S}(f)\right|_{\text {state } 2} E_{P I}(f)\right|_{\text {state 2 }}$, and $\left.E_{F_{2}}(f)\right|_{\text {state } 2}$ are, respectively, calculated contribution of propellant combustion, piston shear, piston impact, and preload release in high preload state $(2 \mathrm{kN})$.

According to (2), the calculated contribution values of every shock source under different frequency based on the explosive bolt of state 1 are shown in Figure 11(a). It indicates that the output shock in overall frequency is driven primarily by preload release in high preload. The contribution of piston shear is mainly at low frequency (below 2,000 Hz) because shear weak surface is a plastic deformation fracture process. In medium frequency $(2,000 \mathrm{~Hz}-7,000 \mathrm{~Hz})$, except for preload release, the piston impact primarily drives the shock output of explosive bolt. The reasons are that the piston impact features a metal-to-metal impact and the piston has an end of travel stop, which plastically deforms. The propellant combustion is not a significant contributor in low and medium frequency band (below 7,000 Hz), but it is greater than piston shear and piston impact in high frequency (above 7,000 Hz) which is always lower than preload release. In addition, it can be found that the ration of propellant combustion in the total event is increasing with frequency, 
TABLE 2: Decoupling analysis results of shock source for explosive bolts.

\begin{tabular}{lcc}
\hline Shock source & $\left.E_{M, S}\right|_{\text {high preload }}(10 \mathrm{kN})$ & $E_{M, S} l_{\text {low preload }}(2 \mathrm{kN})$ \\
\hline Preload release & $57.51 \%$ & $13.15 \%$ \\
Piston impact & $15.01 \%$ & $28.14 \%$ \\
Piston shear & $13.05 \%$ & $29.60 \%$ \\
Propellant combustion & $14.43 \%$ & $29.11 \%$ \\
Total & $100 \%$ & $100 \%$ \\
\hline
\end{tabular}

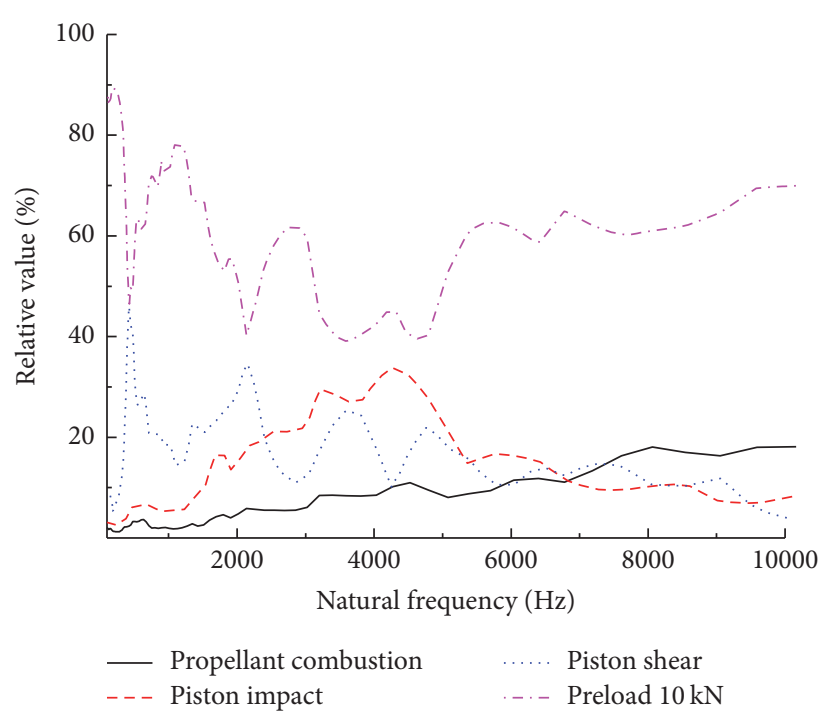

(a) High preload (state 1)

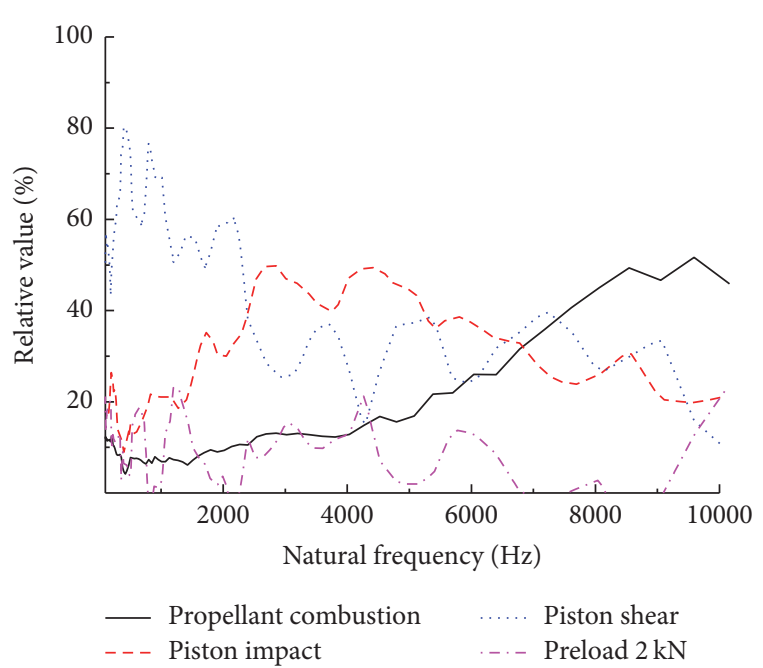

(b) Low preload (state 2)

FIGURE 11: Relative value of output SRS.

which is in accord with the characteristics of combustion shock wave.

In low preload (state 2), according to (3), contribution of every shock source under different frequency ranges is shown in Figure 11(b). It indicates that the preload release is not a significant contributor to the overall shock output of the explosive bolt in low preload, and the other analysis is the same as the high preload.

In order to comprehensively evaluate every shock source's influence, the mean relative values of every shock source in whole frequency domain are calculated as

$$
\left.E_{M, S}\right|_{T}=\frac{\left.\sum_{f=f_{l}}^{f=f_{h}} E_{S}(f)\right|_{T}}{N},
$$

where $\left.E_{M, S}(f)\right|_{T}$ is the mean relative values of every shock source in whole frequency range, $\left.E_{S}(f)\right|_{T}$ is relative contribution values of specific shock source in different frequency, $f_{l}$ and $f_{h}$ are, respectively, the lowest and highest analysis frequency, $N$ represents the number of samples of the frequency vector, and $T$ represents the total SRS of normal explosive bolt with preload.

According to (4), the mean results are shown in Table 2. As Table 2 shows, shock output of shear type explosive bolt in high preload condition is driven primarily by releasing preload; other sources are minor and roughly the same, while they are major contributors in low preload.

\section{Conclusion}

The shock response output from propellant combustion, internal piston shearing weaknesses, internal piston impact, and bolt preload release were decoupled by testing five kinds of explosive bolts with different states. The results show that the characteristics of different shock sources are different, and the shock induced by propellant combustion is mainly caused by high frequency, while piston shear and piston impact are concentrated in medium and low frequency. The preload release is a major contributor to the overall shock response output in high preload condition, accounting for approximately $57.51 \%$, while the others are minor and equivalent. However, in low preload, the contribution of preload release dropped to $13.15 \%$. Without reducing the reliability of the connection and separation, the low shock design of shear type explosive bolts should first take measures from the preload, such as adding a multilayer gasket at the connection surface to dissipate the released prestrain energy, and adding a buffer to absorb the piston impact energy is also beneficial. 


\section{Conflicts of Interest}

The authors declare no conflicts of interest.

\section{Acknowledgments}

This research was sponsored by the NSAF Joint Fund of the National Natural Science Foundation of China and the China Academy of Engineering Physics (Grant no. U1530135).

\section{References}

[1] L. J. Bement and M. L. Schimmel, A Manual for Pyrotechnic Design Development and Qualification, NASA, 1995.

[2] Z. S. Liu, X. J. Wang, X. C. Zhu, and G. H. Wang, Aerospace Pyrotechnic Devices, Chinese Astronautics Press, Beijing, China, 2012.

[3] J. Lee, D. H. Hwang, and J. H. Han, "Numerical study on proshock generation and propagation from pyrotechnic release devices using hydrocodes," in Proceedings of the 22nd International Congress on Sound and Vibration, pp. 1-8, Florence, Italy, 2015.

[4] Y. J. Lee, "The interpretation of separation mechanism of ridgecut explosive bolt using simulation programs," Journal of the Society of Magnetic Resonance in Medicine, vol. 56, no. 2, pp. 395-410, 2004.

[5] Y. Li, The structual, design and numerical analysis of explosive bolt with impair slot, Nanjing University of Science and Technology, Nanjing, China, 2010.

[6] Y. H. Li, X. G. Li, Y. Q. Wen, S. W. Guo, L. Cheng, and H. N. $\mathrm{Mu}$, "Optimal design of an explosive separation device based on LS-DYNA," Journal of Beijing Institute of Technology, vol. 25, pp. 24-28, 2016.

[7] T. Y. Ye, Y. Wang, X. F. Wang, and J. Zhang, "Discussion on the design of underwater explosive bolt," Mine Warfare and Ship Self-Defense, vol. 25, no. 4, pp. 11-14, 2007.

[8] C. T. Hou, W. Yi, T. Tang, K. L. Rong, and B. J. Ma, "Strength study on a typical explosive bolt," Structure and Environment Engineering, vol. 34, no. 4, pp. 32-37, 2011.

[9] C. J. Moening, Pyrotechnic shock flight failures, Institute of environmental sciences pyrotechnic shock tutorial program in 31st Annual Technical Meeting, Washington, DC, USA, 1985.

[10] A. G. Piersol, T. L. Paez, and C. M. Harris, Harris's Shock and Vibration Handbook, McGraw-Hillm, New York, NY, USA, 6th edition edition, 2010.

[11] M. G. Ryschkewitsch, Pyroshock Test Criteria, USA, NASA, 2011.

[12] DOD, Pyroshock, MIL-STD-810G Method 517.1, Department of Defense, New York, NY, USA, 2008.

[13] IEST, Pyroshock Testing Techniques, Institute of Environmental Science and Technology, 2009.

[14] PLA, Laboratory environmental test methods for military materiel-Part 27: Pyroshock test, The Chinese people's liberation army in the equipment department, Beijing, China, 2009.

[15] S. J. Gentz, D. O. Ordway, D. S. Parsons, C. M. Garrison, C. S. Rodgers, and B. W. Collins, Empirical Model Development for Predicting Shock Response on Composite Materials Subjected to Pyroshock Loading, NASA, 2015.

[16] W. O. Hughes and A. M. McNelis, Statistical Analysis of a Large Sample Size Pyroshock Test Data Set Including Post Flight Data Assessment, NASA, 1998.
[17] H. Zhang, T. X. Liu, C. J. Li, S. H. Xiang, and Q. M. Zhang, "Status and application analysis of spacecraft pyroshock protection techniques," Spacecraft Engineering, vol. 23, no. 2, pp. 104-113, 2014.

[18] J. Lee, J.-H. Han, Y. Lee, and H. Lee, "Separation characteristics study of ridge-cut explosive bolts," Aerospace Science and Technology, vol. 39, pp. 153-168, 2014.

[19] J. Lee, J.-H. Han, Y. Lee, and H. Lee, "A parametric study of ridge-cut explosive bolts using hydrocodes," International Journal of Aeronautical and Space Sciences, vol. 16, no. 1, pp. 50-63, 2015.

[20] J. Lee, J. H. Han, and Y. J. Lee, "Design of low-shock ridgecut explosive bolts based on separation behavior analysis," in Proceedings of the 40th International Pyrotechnics Seminar, Colorado Springs, Colo, USA, 2014.

[21] J. Lee, D.-H. Hwang, J.-K. Jang et al., "Pyroshock prediction of ridge-cut explosive bolts using hydrocodes," Shock and Vibration, vol. 2016, Article ID 1218767, 2016.

[22] H. J. Huang, J. P. Wang, Y. J. Mao, X. H. Yue, and J. Lv, "Influence of pretightening force of explosive bolts on impulse response," Journal of Vibration and Shock, vol. 34, no. 16, pp. 166-169, 2015.

[23] Y. K. Ye and N. Yan, "Study on technology to low separation impact of pyrotechnic separator," Initiators and Pyrotechnics, vol. 1, pp. 13-16, 2011.

[24] H. L. Liu, D. L. Cui, and S. Z. Yan, "Numerical simulation of the dynamic fracture of non-contamination explosive bolts," Journal of Tsinghua University (Science and Technology), vol. 55, no. 3, pp. 292-297, 2015.

[25] J.-R. Lee, C. C. Chia, and C.-W. Kong, "Review of pyroshock wave measurement and simulation for space systems," Measurement, vol. 45, no. 4, pp. 631-642, 2012. 


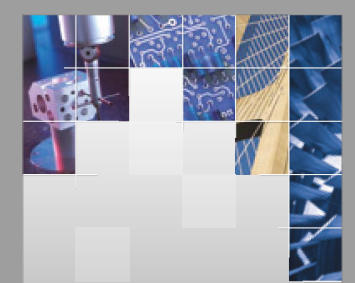

\section{Enfincering}
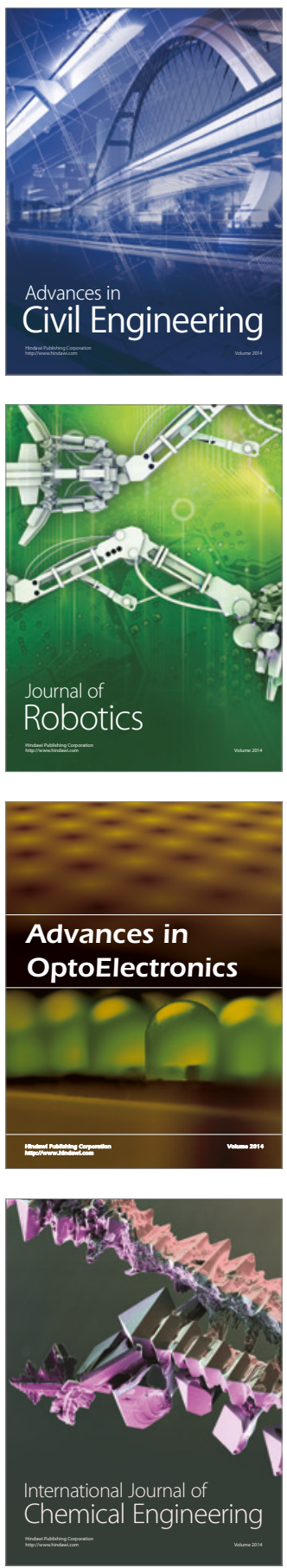

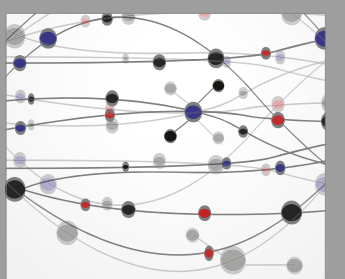

The Scientific World Journal

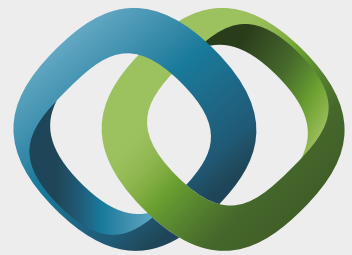

\section{Hindawi}

Submit your manuscripts at

https://www.hindawi.com
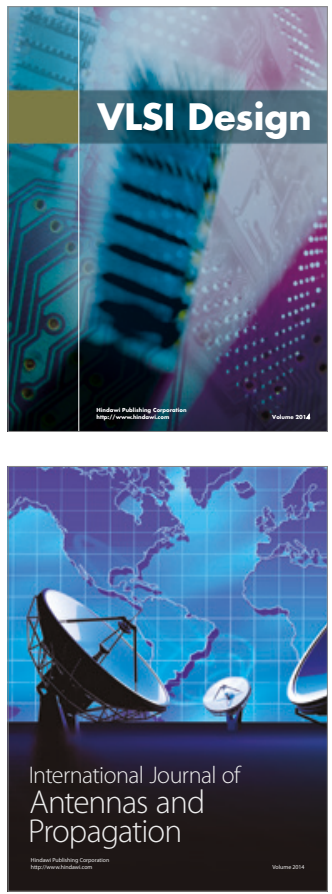

\section{Rotating}

Machinery
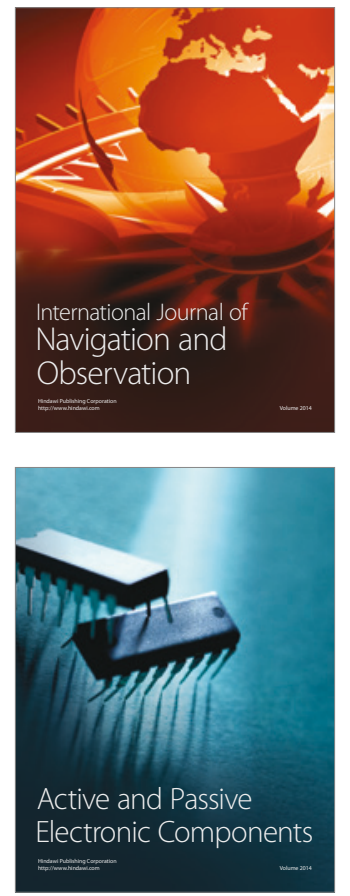
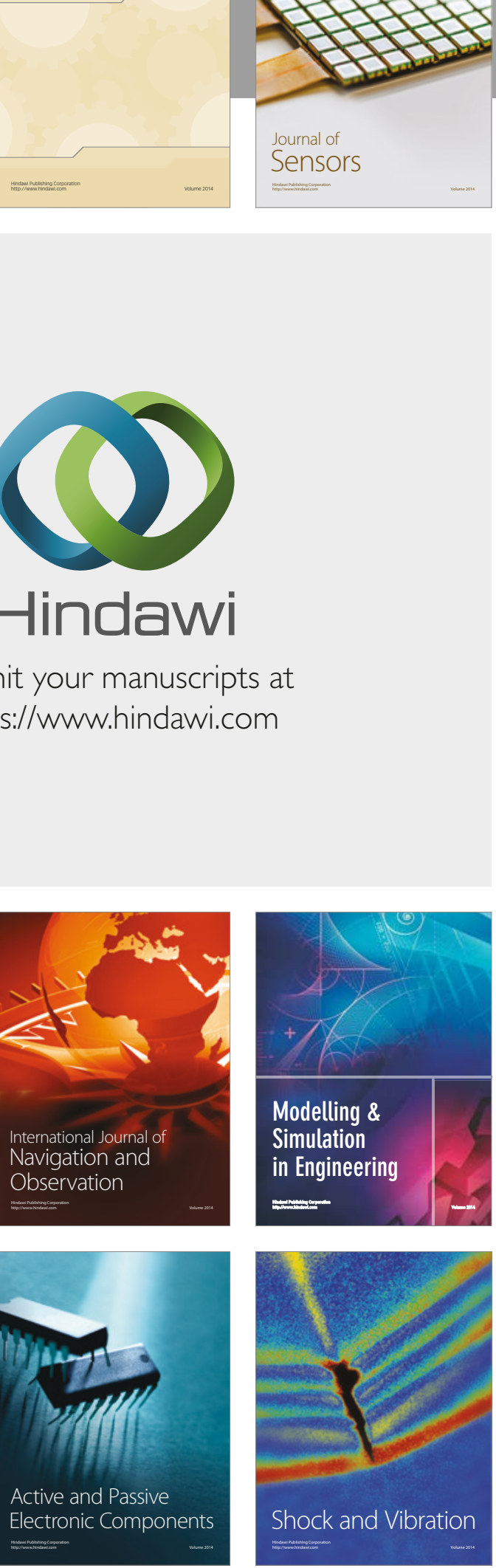
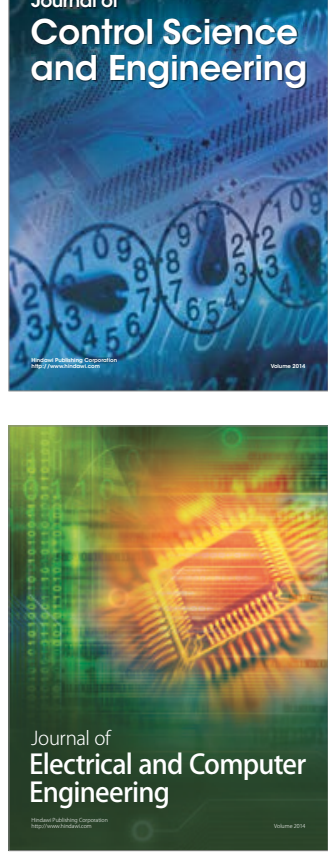

Distributed

Journal of

Control Science

and Engineering
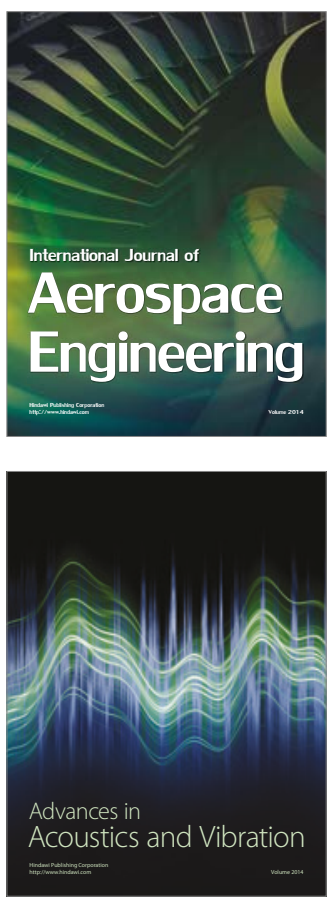

Sensor Networks 